Instituto Internacional de Investigación y Desarrollo Tecnológico Educativo INDTEC, C.A.

DOI: https://doi.org/10.29394/Scientific.issn.2542-2987.2020.5.18.13.254-275

OAI-PMH: http://www.indteca.com/ojs/index.php/Revista Scientific/oai

Ensayo Original / Original Essay

\title{
Características de un Docente Innovador: Siete Claves para Una Buena Práctica Docente
}

Autores: Christian Virgilio Guerrero Salazar Unidad Educativa Bilingüe Torremar, TORREMAR chguerrero.ascaped@gmail.com

Guayaquil, Ecuador https://orcid.org/0000-0003-3108-1846

Yeimer Prieto López Universidad Espíritu Santo, UEES yeiprietolopez@gmail.com

Guayaquil, Ecuador https://orcid.org/0000-0001-7369-1402

\section{Resumen}

La realidad educativa contemporánea dista mucho de la que conocimos la gran mayoría de los que nos dedicamos a la docencia desde hace más de quince años. Desde las disfunciones familiares, pasando por los cambios sociales, ideológicos, tecnológicos y científicos hasta llegar a la infinidad de trastornos y necesidades educativas especiales, que se presentan en los estudiantes actuales, esta realidad exige del docente una preparación cada vez más idónea que le permita desarrollarse en la profesión con mejores posibilidades de éxito. Los cambios nunca han sido fáciles; es por eso que el docente necesita en primer lugar realizar una transformación de sus propios paradigmas mentales, que le permita desarraigar las viejas prácticas pedagógicas innecesarias o desactualizadas a través de la formación e investigación educativa constante. El presente ensayo busca proponer sobre la base de la investigación educativa, una síntesis de las principales características que permiten al docente contemporáneo enfrentar los cambios educativos de una manera coherente y eficaz, utilizando técnicas y herramientas pedagógicas actualizadas, basadas en el conocimiento profundo de teorías educativas y el uso de las tecnologías.

Palabras clave: innovación educativa; formación docente; proceso enseñanza-aprendizaje.

\section{Cómo citar este ensayo:}

Guerrero, C., \& Prieto, Y. (2020). Características de un Docente Innovador: Siete Claves para Una Buena Práctica Docente. Revista Scientific, 5(18), 254-275, e-ISSN: 2542-2987. Recuperado de: https://doi.org/10.29394/Scientific.issn.2542-2987.2020.5.18.13.254-275

Fecha de Recepción:

01-06-2020
Fecha de Aceptación:

24-09-2020
Fecha de Publicación: 05-11-2020 


\title{
Characteristics of an Innovative Teacher: Seven Keys to Good Teaching Practice
}

\begin{abstract}
The contemporary educational reality is far from the one known to the vast majority of us who have dedicated ourselves to teaching for more than fifteen years. From family dysfunctions, through social, ideological, technological and scientific changes to the infinity of disorders and special educational needs that occur in current students, this reality demands from the teacher an increasingly suitable preparation that allows develop in the profession with better chances of success. Changes have never been easy; That is why the teacher needs first of all to carry out a transformation of their own mental paradigms, which allows them to uproot the old unnecessary or outdated pedagogical practices through training and constant educational research. This essay seeks to propose on the basis of educational research, a synthesis of the main characteristics that allow contemporary teachers to face educational changes in a coherent and effective way, using updated pedagogical techniques and tools, based on deep knowledge of educational theories. and the use of technologies.
\end{abstract}

Keywords: educational innovation; teacher training; teaching learning process.

\footnotetext{
How to cite this essay:

Guerrero, C., \& Prieto, Y. (2020). Characteristics of an Innovative Teacher: Seven Keys to Good Teaching Practice. Revista Scientific, 5(18), 254-275, e-ISSN: 2542-2987. Recovered from: https://doi.org/10.29394/Scientific.issn.2542-2987.2020.5.18.13.254-275
}

Date Received: 01-06-2020
Date Acceptance:

24-09-2020
Date Publication:

05-11-2020 


\section{Introducción}

La innovación es hoy en día uno de los pilares que sostienen a la calidad educativa en todos los ámbitos de la enseñanza, y de acuerdo a Díaz y Guambi (2018): "constituye el motor impulsor del desarrollo y crecimiento empresarial hacia la competitividad en un mercado cada vez más exigente y demandante" (pág. 212); haciendo que se vuelva urgente un cambio paradigmático que permita a los docentes visualizar el camino a seguir hacia mejores propuestas de aula.

A través de este estudio, se pretende hacer una reflexión consciente acerca de la innovación educativa y algunas claves que se consideran fundamentales tanto en el conocimiento didáctico-científico, como en la práctica docente. La investigación se basa en un estudio bibliográfico de carácter descriptivo, fundamentado en las propuestas y teorías de diferentes autores y organismos internacionales, que ayudan a comprender la realidad educativa contemporánea, así como las técnicas y estrategias metodológicas que se requieren para enfrentar los retos educativos que se presentan actualmente.

El presente ensayo muestra los esfuerzos de los investigadores, por incrementar la generación de conocimientos y el desarrollo de la innovación pedagógica y tecnológica en el proceso áulico, los hallazgos muestran procesos de innovación informales, no planeados, en muchos casos orientados a la imitación, estrategias repetitivas que adolecen de la innovación y nuevas aportaciones científicas. En lo metodológico se observa énfasis en trabajos empíricos con incipientes desarrollos de tipo explicativo y limitado uso de técnicas de análisis cualitativo y cuantitativo que puedan producir un verdadero impacto en el aprendizaje.

Cabe recalcar en este punto, que no existe una receta mágica que permita al docente la consecución de buenas prácticas educativas de una manera ideal -entiéndase constante y perfecta- ya que inclusive existen aún 
grandes divergencias entre distintos pensadores acerca de una u otra característica esencial; sino más bien debe entenderse como sinónimo de buena práctica docente a la búsqueda constante de herramientas y desafíos que a través de la experiencia y el ensayo-error, vayan creando en el educador un eclecticismo valioso a la hora de innovar en el aula.

\section{Desarrollo}

Las características que definen la calidad pedagógica de un profesor pueden llegar a ser innumerables tomando en cuenta las diferencias que como seres humanos podemos llegar a tener, pero a través de este estudio vamos a enfocarnos en siete aspectos que, a consideración propia y también de distintos autores referenciados en el desarrollo del escrito, podrían ser las claves más relevantes para una buena práctica docente. Para el Ministerio de Educación del Perú (2014), los maestros que realizan buenas prácticas docentes:

[...] Comparten como objetivo común el lograr un buen aprendizaje en sus alumnos y se encuentran en constante descubrimiento de su quehacer profesional, lo que les permite alejarse de las prácticas rutinarias y generar nuevas actividades de aprendizaje gracias al fortalecimiento de la autoconfianza en sus alumnos y pares y el desarrollo de sus potencialidades [...] (pág. 16).

A continuación, trataremos de describir de una forma concisa y clara estos siete aspectos relevantes, que todo maestro que pretende innovar su práctica educativa debe tener en consideración al momento de diseñar y ejecutar sus clases, como se muestra en la figura 1. 
Figura 1. Siete claves para una buena práctica docente.

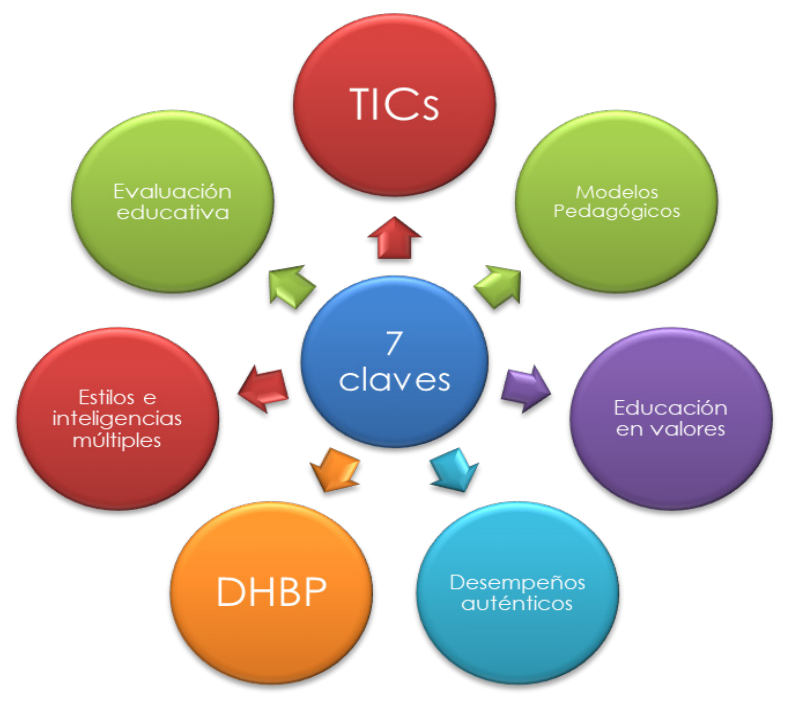

Fuente: Los Autores (2020).

Manejo y aplicación de las Tecnologías de la información y comunicación (TIC), es considerado hoy en día como un aspecto clave para la innovación educativa. Es una herramienta fundamental para poner en práctica diversas metodologías y técnicas de enseñanza, desarrollándose desde distintos modelos como el Aula Invertida, el Aprendizaje móvil, la Educación en línea, entre otros que van surgiendo.

A diferencia del siglo pasado, en el que la educación se movía a través de certezas y objetividades, la nueva era trajo consigo avances tecnológicos que cada vez se van haciendo más profundos, de tal manera que, en la nueva sociedad del conocimiento es imperante el uso de la tecnología para el manejo de la información como medio circulante que vino a reemplazar a la industria en su rol de valor primordial de la transformación social. Algunas de sus ventajas son precisamente la flexibilidad de uso y el acceso cada vez más rápido y sencillo que éstas tienen, entre otras ventajas que se enuncian en la figura 2. 


\section{Ensayo Original / Original Essay}

Figura 2. Ventajas de las TIC.

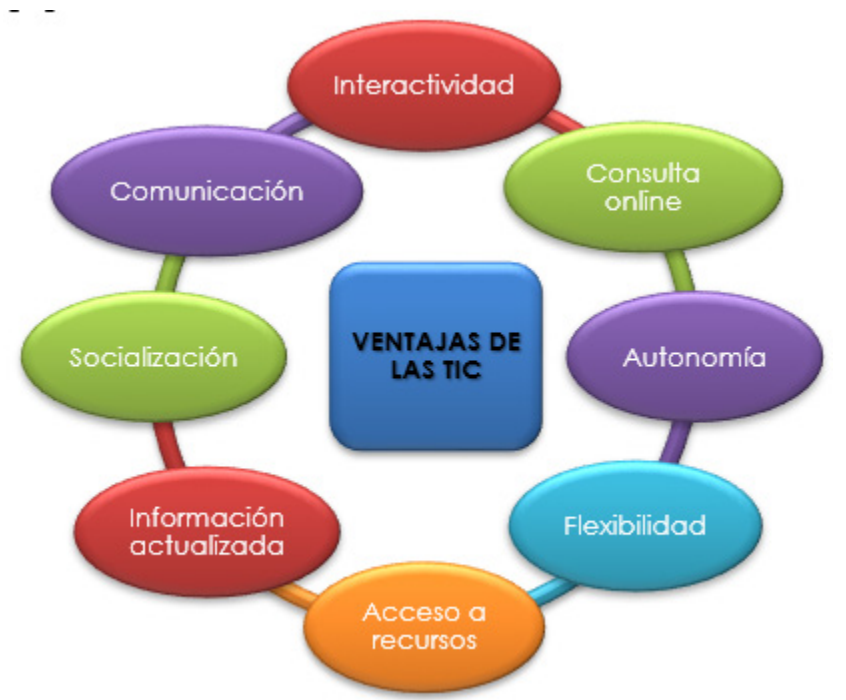

Fuente: Los Autores (2020); Adaptación de Ruiz y Tesouro (2013).

Es por esto que el uso de las nuevas tecnologías se hace tan imperante para el profesor, convirtiéndose en una herramienta motivacional para que los estudiantes -nativos digitales- vean la educación de una manera distinta, y a la vez cuenten con recursos audiovisuales que permitan el uso de todos sus sentidos para una mejor comprensión de los conceptos estudiados. De acuerdo a Castro, Guzmán y Casado (2007):

Las TIC, son cada vez más amigables, accesibles, adaptables herramientas que las escuelas asumen y actúan sobre el rendimiento personal y organizacional. Estas escuelas que incorporan la computadora con el propósito de hacer cambios pedagógicos en la enseñanza tradicional hacia un aprendizaje más constructivo (pág. 217).

Uso de modelos pedagógicos adecuados y contextualizados, para el desarrollo eficaz de las acciones educativas del centro. Esta implementación requiere políticas institucionales que determinen un camino a seguir de manera conjunta por todo el profesorado, para la unificación de criterios que a largo plazo permita el mejoramiento en la calidad de la enseñanza. 
Los mejores sistemas educativos del mundo han apostado por la innovación constante a la luz de teorías y modelos tanto tradicionales como vanguardistas que les permitan desarrollar un eclecticismo pedagógico que se adapte a las realidades particulares de cada país e inclusive de cada institución. Existen diversos modelos pedagógicos, desde los tradicionales hasta los actuales o conocidos como la Escuela nueva de la educación, que integran además de teorías pedagógicas efectivas, el uso de herramientas didácticas contemporáneas como algunas de las nombradas en el apartado anterior.

Pero ¿por qué es importante la utilización de modelos pedagógicos?, a saber, porque brindan un delineamiento metodológico unificado, el cual permite que los docentes se guíen por hilos conductores comunes, sin dejar de impregnarle a cada asignatura los principios didácticos propios de su naturaleza científica.

Estos nuevos modelos deben también brindar posibilidades de cambio e innovación respecto a los tradicionales, que permitan ejecutar roles más activos en los procesos de enseñanza-aprendizaje. Procesos como la articulación de saberes previos, el uso de la vida real como detonante de aprendizaje, el diseño de situaciones problémicas del campo profesional o de interés para el estudiante, el uso de técnicas de aprendizaje cooperativo y colaborativo, el desarrollo de la reflexividad en los estudiantes, entre otros, deben formar parte inherente de cualquier modelo que pretenda hoy en día un desarrollo adecuado de las capacidades y competencias académicas de los alumnos.

Educar en valores, para una verdadera transformación humanista de las nuevas generaciones. En un mundo tan cambiante y acelerado, en el que cada vez más se incrementa la violencia, la intolerancia y prima el interés propio sobre el bien común; es esencial para la escuela, que los docentes se comprometan profundamente en la educación en valores en rescate de la 
sensibilización de nuestros alumnos ante los males que aquejan a la humanidad. Como lo describe Del Salto (2015):

Los valores universales de verdad, justicia, respeto, humanismo, solidaridad, libertad, responsabilidad, igualdad, fraternidad, solidaridad, equidad, paz, bondad, honradez y honestidad; completan la formación de la persona en el sentido de un estado de paz y armonía. Estos valores están presentes desde los inicios de la humanidad, y su transmisión ha sido una preocupación constante de todas las culturas, a la vez que constituye una de las bases sobre la cual la ética y la moral se fortalecen en cualquier campo y época (pág. 5019).

Por tanto, el educador actual no puede concentrarse solamente en la formación académica de sus estudiantes, sino que debe ser una fuente de la cual sus alumnos puedan alimentarse de aquellos valores que necesita para su formación integral. Pero, nadie puede dar lo que no tiene, así que el docente como espejo en el que se reflejarán sus estudiantes debe ser el primero en dar ejemplo de puntualidad, respeto y ética profesional, pues como reza una frase atribuida al gran Albert Einstein (1879-1955): «Dar ejemplo no es la principal manera de influir sobre los demás. Es la única manera».

Estrategias metodológicas como desempeños auténticos (o al revés). Un maestro que pretende hacer cambios importantes en la educación para lograr dejar huellas perennes en la vida de sus estudiantes no puede darse el lujo de continuar utilizando las mismas estrategias metodológicas que se usaban en el siglo pasado. La repetitividad de las acciones, está demostrado que genera hábitos, los cuales son necesarios e importantes para la educación, pero no deben ser los únicos recursos utilizados por el docente.

Es claro que en el aprendizaje de asignaturas de ciencias y matemáticas se torna esencial -sin que por esto no sea relevante en las otrasla práctica constante de ejercicios modelados para procesos algorítmicos estructurados; pero se requiere avanzar más allá si no queremos quedarnos en un nivel netamente transferencial del desarrollo del pensamiento, como se 
describe en el modelo de Congruencia Educativa de Córdova, Oliveros y Villena (2013): sino llegar hacia los niveles más altos como el crítico y el creativo. Según Córdova, Peña y Sarzosa (2012): en el nivel crítico "se concentran las competencias de tipo analítico, las mismas que permiten conjugar las competencias reproductivas y transferenciales para obtener resultados fundamentados" (pág. 53); mientras que en el nivel creativo "se manejan competencias que permiten desarrollar innovaciones, modelos originales y que explotan la creatividad dentro de una misma área de trabajo" (pág. 53).

Para lograr avanzar entonces hacia esos altos niveles de desarrollo cognitivo que pretendemos, se hace urgente la utilización de procesos de clase en los que abunden actividades generadoras de aprendizaje efectivo, que vayan de la mano con los intereses actuales de los estudiantes y los enfrenten a situaciones problémicas que pongan a prueba su comprensión de los conceptos estudiados; lo que desde el año 1967 el grupo de investigadores del Proyecto Zero de Harvard denominó "Desempeños auténticos». De acuerdo con Gardner (2004), citado por el Ministerio de Educación del Ecuador (2011):

Propone la necesidad de encaminar a quienes aprenden hacia la comprensión de diversas formas de pensamiento disciplinar que exploren con profundidad temas esenciales de cada disciplina, de modo que se acerquen a cómo piensa y cómo actúa un experto para utilizar estas formas de pensamiento y acción en la comprensión de su propio mundo (pág. 153).

Este tipo de actividades requieren de parte del maestro una profundización de sus conocimientos acerca de los intereses de los estudiantes, así como un espíritu investigativo que lo mueva constantemente hacia la búsqueda de nuevas ideas para contextualizar hacia la realidad lo mejor posible sus estrategias metodológicas.

Desarrollo de habilidades básicas del pensamiento, como pilar 
fundamental para el posterior desarrollo de las habilidades de orden superior. En lo referente a este tema existe cierta confusión, ya que diversos autores llaman de igual manera a distintas propuestas de habilidades cognitivas. Así, algunos les llaman Procesos cognitivos básicos, y otros Destrezas intelectivas, pero lo realmente importante es reconocer que estas habilidades son esencialmente fundamentales para la concreción de los diferentes ciclos de aprendizaje que se pretendan aplicar en el aula. En otras palabras, está demostrado que, si no se completa este proceso, muy probablemente el aprendizaje no se ha arraigado completamente en la mente del alumno.

El proceso de aplicación de estas habilidades es sistemático y le brinda coherencia cognitiva a la estructura didáctica de las clases, permitiendo una mejor conexión entre los diferentes componentes del ciclo de aprendizaje que el docente aplique en su asignatura. Iniciar con la observación de fenómenos de la realidad para entender el concepto a estudiar, pasando por la descripción, comparación, clasificación desde distintos criterios taxonómicos, análisis estructural de los componentes teóricos de un concepto, para a través de la síntesis integrarlos en un todo permitiendo establecer su funcionamiento conjunto, hasta llegar a la evaluación que debe desarrollarse en sus distintas modalidades -hetero, co y auto- para permitirle al estudiante la capacidad de cuestionar y cuestionarse, determinando a través de juicios de valor la idoneidad del camino seguido en la consecución de los aprendizajes.

Una vez que las habilidades básicas mostradas en la figura 3, logran desarrollarse coherentemente a través del proceso de enseñanza y aprendizaje de cualquier asignatura, el maestro debe ser capaz de llevar a sus estudiantes a la reflexión profunda acerca de las etapas por las que se desenvolvió en el camino hacia la comprensión, esto es la Metacognición. 


\section{Ensayo Original / Original Essay}

Figura 3. Habilidades básicas del pensamiento.

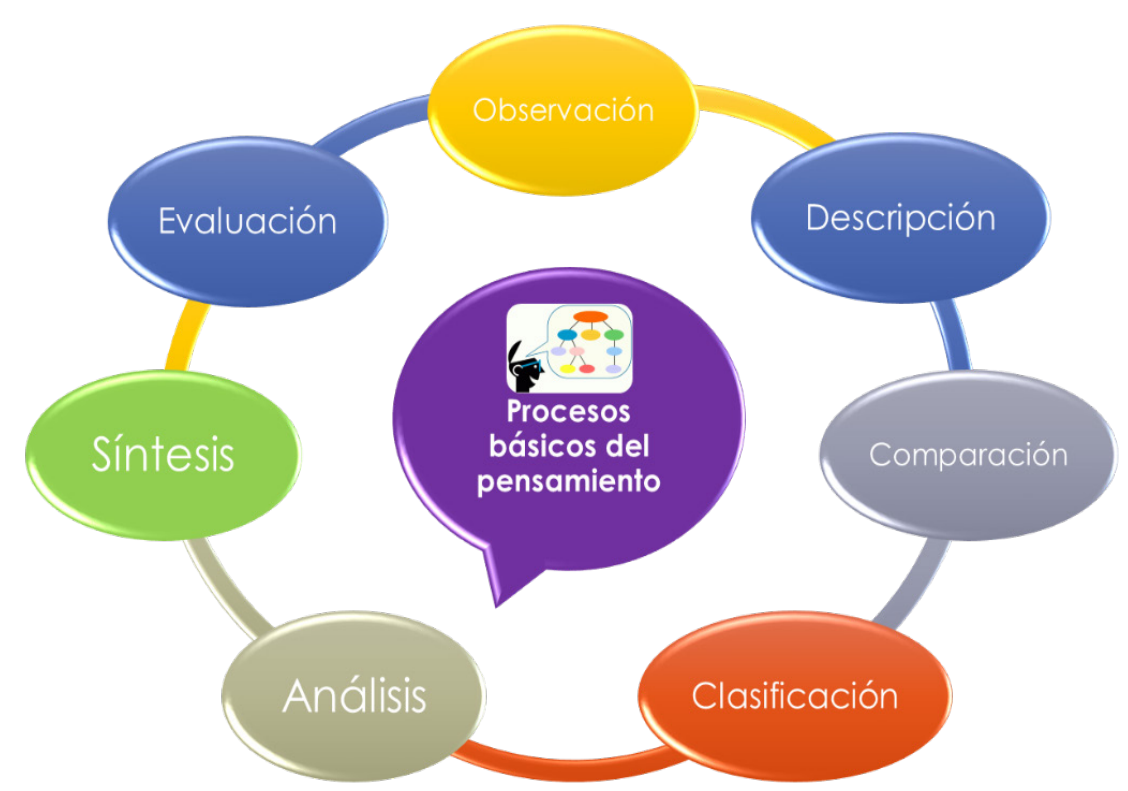

Fuente: Los Autores (2020).

Para Vázquez-Chaves (2015): "[...] la metacognición potencia la capacidad de las personas para conocer, controlar y evaluar sus propios procesos, mediante la creación e implementación de estrategias metacognitivas que permiten derribar las barreras de aprendizaje, así como las actitudinales y las de participación" (págs. 15-16).

Es por esto, que el docente debe diseñar estrategias metodológicas que le permitan a los estudiantes realizar un análisis profundo de sus propias capacidades, para poder entender desde la experiencia de aprendizaje, sus propias maneras de llegar a la comprensión, pues como indica Flavel (1979), citado por García, Rodríguez, González-Castro, Álvarez-García y GonzálezPienda (2016): es importante para los estudiantes "[...] diferenciar aquellas estrategias que son útiles para ellos, sabiendo reconocer porqué, cómo y cuándo aplicarlas" (pág. 474); estableciendo de esta manera procesos mentales que a largo plazo le permitirán crear sus propias técnicas para llegar al conocimiento. 
Estilos de aprendizaje e inteligencias múltiples. Un común denominador de las clases de Matemática del mejor profesor que tuve en el colegio, era el dictar el problema mientras lo iba escribiendo en la pizarra para que los estudiantes lo copiáramos; no conforme con esto, luego se daba la vuelta hacia nosotros y nos ejemplificaba el problema tomando incluso en ocasiones como actores a los compañeros de las primeras bancas para dramatizar la situación.

Pasaron los años y sentí la vocación de convertirme también en profesor de Física y Matemática, y estoy seguro que la excelente didáctica de este maestro fue uno de los puntos clave en mi decisión profesional; pero tuvieron que pasar muchos años más para que a través del estudio y la investigación pudiera entender las razones pedagógicas que motivaron a este buen docente a hacer esa rutina común en cada resolución de problemas.

$Y$ es que, el conocimiento de los diferentes estilos de aprendizaje que tiene el ser humano, resulta clave para una mejor planificación microcurricular, pues nos permite diseñar un proceso que no solamente use las Tecnologías de la información y la comunicación (TIC), eduque en valores, use modelos pedagógicos contemporáneos y tenga coherencia en el desarrollo de habilidades del pensamiento a través de desempeños auténticos, sino que sistematizará todos estos aspectos integralmente de manera que pueda llegar no solo a un pequeño porcentaje de la población estudiantil sino a la gran mayoría, tomando en cuenta todos los estilos de aprendizaje posibles así como los diferentes tipos de inteligencias que pudieran tener como predominantes.

En lo referente a los estilos de aprendizaje, el modelo de Programación Neurolingüística PNL de Bander y Glinder, establece la existencia de 3 esenciales: visual, auditivo y kinestésico, cuyas características se muestran en el cuadro 1 lo siguiente: 
Instituto Internacional de Investigación y Desarrollo Tecnológico Educativo INDTEC, C.A.

DOI: https://doi.org/10.29394/Scientific.issn.2542-2987.2020.5.18.13.254-275

OAI-PMH: http://www.indteca.com/ojs/index.php/Revista_Scientific/oai

Ensayo Original / Original Essay

Cuadro 1. Características de los estilos de aprendizaje según PNL.

\begin{tabular}{|c|c|c|c|}
\hline & VISUAL & AUDITIVO & KINESTÉSICO \\
\hline Conducta & $\begin{array}{l}\text { Organizado, } \\
\text { ordenado, } \\
\text { observador y } \\
\text { tranquilo. } \\
\text { Preocupado por } \\
\text { su aspecto. Voz } \\
\text { aguda, barbilla } \\
\text { levantada. } \\
\text { Se le ven las } \\
\text { emociones en la } \\
\text { cara. }\end{array}$ & $\begin{array}{l}\text { Habla solo, se distrae } \\
\text { fácilmente. Mueve los } \\
\text { labios al leer. Facilidad } \\
\text { de palabra, no le } \\
\text { preocupa especialmente } \\
\text { su aspecto. Monopoliza } \\
\text { la conversación. Le } \\
\text { gusta la música. Modula } \\
\text { el tono y timbre de voz. } \\
\text { Expresa sus emociones } \\
\text { verbalmente. }\end{array}$ & $\begin{array}{c}\text { Responde a las } \\
\text { muestras físicas de } \\
\text { cariño, le gusta tocarlo } \\
\text { todo, se mueve y gesticula } \\
\text { mucho. Sale bien arreglado } \\
\text { de casa, pero enseguida se } \\
\text { arruga porque no para. } \\
\text { Tono de voz más bajo, } \\
\text { porque habla alto con la } \\
\text { barbilla hacia abajo. } \\
\text { Expresa sus emociones } \\
\text { con movimientos. }\end{array}$ \\
\hline Aprendizaje & $\begin{array}{c}\text { Aprende lo que } \\
\text { ve. Necesita una } \\
\text { visión detallada y } \\
\text { saber a dónde } \\
\text { va. Le cuesta } \\
\text { recordar lo que } \\
\text { oye. }\end{array}$ & $\begin{array}{c}\text { Aprende lo que oye, a } \\
\text { base de repetirse a sí } \\
\text { mismo paso a paso } \\
\text { todo el proceso. Si se } \\
\text { olvida de un solo paso } \\
\text { se pierde. No tiene una } \\
\text { visión global. }\end{array}$ & $\begin{array}{c}\text { Aprende lo que } \\
\text { experimenta } \\
\text { directamente, aquello } \\
\text { que involucre } \\
\text { movimiento. Le cuesta } \\
\text { comprender lo que no } \\
\text { puede poner en } \\
\text { práctica. }\end{array}$ \\
\hline
\end{tabular}

Fuente: Cisneros (2004).

Este modelo, conocido comúnmente como el VAK por la unión de las 3 iniciales, considera que tenemos tres formas generales de representación mental de la información adquirida, lo cual se demuestra en las formas cotidianas que tenemos para acceder a la información a través de los sentidos.

De acuerdo al manual de estilos de aprendizaje desarrollado por la Subsecretaria de Educación Media Superior, Cisneros (2004), presenta que:

Utilizamos el sistema de representación visual siempre que recordamos imágenes abstractas (como letras y números) y concretas. El sistema de representación auditivo es el que nos permite oír en nuestra mente voces, sonidos, música. Cuando recordamos una melodía o una conversación, o cuando reconocemos la voz de la persona que nos habla por teléfono estamos utilizando el sistema de representación auditivo. Por último, cuando recordamos el sabor de nuestra comida favorita, o lo que sentimos al escuchar una canción estamos utilizando el sistema de representación kinestésico [...] (pág. 30). 
La consideración de que estos estilos están presentes necesariamente en nuestros salones de clase, permite al docente el diseño de una mayor diversidad de desempeños auténticos, que sean capaces de abarcar las diferentes formas de representación mental asegurando en cierta medida un mayor porcentaje de logros en los resultados de la comprensión.

El otro aspecto clave a considerar en este punto es el de que la inteligencia no es ya concebida bajo los criterios unitarios y sesgados que anteriormente provocaban su constante confusión con el coeficiente intelectual, sino que, a partir de los estudios de Gardner (2015): hoy podemos claramente evidenciar que existen por lo menos 8 diferentes tipos de inteligencias como se muestra en el cuadro 2.

Cuadro 2. Descripción de los 8 tipos de inteligencia.

Inteligencia Lógico-Matemática: utilizada para resolver problemas de lógica y matemáticas. Es la inteligencia que tienen los científicos. Se corresponde con el modo de pensamiento del hemisferio lógico y con lo que la cultura ha considerado siempre como la única inteligencia.

Inteligencia Lingüística: la que tienen los escritores, los poetas, los buenos redactores. Utiliza ambos hemisferios.

Inteligencia Espacial: consiste en formar un modelo mental del mundo en tres dimensiones. Es la inteligencia que tienen los marineros, los ingenieros, los cirujanos, los escultores, los arquitectos, o los decoradores.

Inteligencia Musical: es naturalmente la de los cantantes, compositores, músicos, bailarines.

Inteligencia Corporal-kinestésica o la capacidad de utilizar el propio cuerpo para realizar actividades o resolver problemas. Es la inteligencia de los deportistas, los artesanos, los cirujanos y los bailarines.

Inteligencia Intrapersonal: es la que nos permite entendernos a nosotros mismos. No está asociada a ninguna actividad concreta.

Inteligencia Interpersonal: la que nos permite entender a los demás, y la solemos encontrar en los buenos vendedores, políticos, profesores o terapeutas. La inteligencia intrapersonal y la interpersonal conforman la inteligencia emocional y juntas determinan nuestra capacidad de dirigir nuestra propia vida de manera satisfactoria

Inteligencia Naturalista: la que utilizamos cuando observamos y estudiamos la naturaleza. Es la que demuestran los biólogos o los herbolarios.

Fuente: Suárez, Maiz y Meza (2010). 
Esta teoría permite enriquecer el conocimiento acerca de las razones por las cuales no todos los alumnos son capaces de realizar ciertas actividades, entendiendo por fin el hecho real de que las dificultades de aprendizaje no tienen origen necesariamente en la poca capacidad o la irresponsabilidad del estudiantado; sino que nos invita por el contrario a repensar lo pedagógico en la búsqueda de mejores herramientas para la selección y manejo de los desempeños de aula con los que podemos trabajar de forma globalizada los diferentes tipos de inteligencia, logrando un desarrollo integral de todos y realzando a la vez individualmente los predominantes.

En términos sencillos, el maestro debe ser capaz de diseñar clases variadas con actividades de aprendizaje que abarquen paulatinamente a todos los tipos de inteligencia, permitiendo su desarrollo eficaz a través de la motivación constante y desempeños que potencien sus intereses científicos.

Evaluación educativa permanente, sistemática, científica y diversa. Esta última clave, cierra de forma coherente esta propuesta pedagógica, que a manera de ensayo pretende reflexionar a través de argumentos científicopedagógicos, los conocimientos y experiencias básicas que el docente contemporáneo requiere a la luz de los nuevos cambios generacionales.

En este sentido, Fernández (2005): define a la evaluación como el "proceso contextualizado y sistematizado, intencionalmente diseñado y técnicamente fundamentado, de recopilación de información relevante, fiable, y válida, que permita emitir un juicio valorativo en función de los criterios previamente determinados como base para la toma de decisiones" (pág. 35). El Reglamento General a la Ley Orgánica de Educación Intercultural (LOEI), promulgado por el Ministerio de Educación (2012):

La evaluación estudiantil es un proceso continuo de observación, valoración y registro de información que evidencia el logro de objetivos de aprendizaje de los estudiantes y que incluye sistemas de retroalimentación, dirigidos a mejorar la metodología de enseñanza y los resultados de aprendizaje (art. 
184).

Esto implica que la evaluación educativa debe poseer características intrínsecas muy bien definidas, que determinen que sus métodos sean realmente efectivos en la consecución de evidencias que nos permitan realizar juicios de valor adecuados. De acuerdo con Alves y Acevedo (2002), citado por Figueroa (2015): "plantean que la evaluación educativa debe ser entendida como una actividad sistemática y continua, integrada a la realidad educativa, para conocerla, mejorarla y valorarla de forma que ayude y oriente a los participantes" (pág. 311). Cada una de las características que se establecen en la ley, le brindan a la evaluación educativa una capacidad profunda de análisis que sirve a corto y largo plazo para convertir los procesos evaluativos en herramientas de juicio más confiables, objetivas y válidas.

Es permanente, pues se requiere la constante comparación cronológica y evolutiva del desarrollo cognitivo y formativo de los estudiantes. Esta característica le confiere a la evaluación su dimensión retro alimentadora, ya que su continuidad permite hacer los cambios necesarios, para modificar aquellos aspectos que sean susceptibles de mejora.

Es sistemática, ya que responde a un plan previamente elaborado y paralelamente Navarro, Falconí y Espinoza (2017): "organiza y desarrolla instrumentos válidos y confiables para la obtención de información relevante" (pág. 62). Por último, se considera científica, desde el punto del uso de técnicas e instrumentos adecuados y coherentes que debe utilizar para la consecución de los resultados y la contrastación de los mismos con los objetivos planteados.

Además de las características nombradas se debe recalcar que, en la actualidad también se torna imperante diversificar e individualizar los procesos para lograr una mayor objetividad y justicia en este aspecto, tradicionalmente tratado de forma generalizada sin tomar en cuenta las diferencias individuales. 
En esta línea, el uso de las TICs se vuelve muy valioso. A criterio de Tourón, Santiago y Díez (2014):

Para que la individualización sea una realidad que permita un progreso más personalizado a cada alumno respecto al currículo, y para que la evaluación se convierta en el mecanismo necesario que oriente este progreso, es imprescindible recurrir a la tecnología digital (pág. 12).

Todos estos aspectos de la educación, deben ser conocidos profundamente por el educador, de manera que no descuide ninguno de ellos al momento de diseñar sus instrumentos en concordancia con las diferentes técnicas de evaluación conocidas, sin caer en lo rutinario y buscando variar continuamente las formas de evaluación en el aula de clases.

\section{Conclusiones}

Como hemos podido evidenciar, la integración de estos siete aspectos claves, es inherente, y se vinculan de forma natural de tal manera que tarde o temprano, incluso quienes nuca se han puesto a reflexionar sobre el accionar educativo, llegarán a las mismas conclusiones que este escrito propone.

El análisis realizado también permite concluir, que se requiere un cambio cultural que nos exija como profesores, acostumbrarnos a la lectura, al estudio y la preparación constante, de la mano con la investigación científica; para romper con el estatismo y la conformidad que parecen haberse vuelto parte de nuestra cotidianidad.

Es por esto que se torna imperante la creación de políticas que fortalezcan la capacitación de los maestros de forma gratuita, o al menos con un costo acorde a los sueldos establecidos, con el fin de crear una cultura de la actualización y la investigación entre los docentes de todos los niveles.

No hay excusas para el cambio, ya que podemos afirmar con base en las experiencias analizadas que la innovación es aplicable a cualquier 
institución educativa, solo basta tener la iniciativa firme de proveer el clima y los recursos necesarios -tanto físicos como humanos- que permitan estructurar un plan de acción basado en las claves propuestas y otras más que puedan complementar el camino para llevar a cabo los procesos ideales que cada entorno comunitario necesita.

La innovación educativa puede convertirse en una realidad, solo en la medida que los docentes estén dispuestos a cambiar sus paradigmas mentales y lanzarse a la búsqueda de mejores opciones pedagógicas con un espíritu científico, que le permita, en la grandeza de su vocación actualizarse constantemente por el bien de su alumnado.

\section{Referencias}

Castro, S., Guzmán, B., \& Casado, D. (2007). Las Tic en los procesos de enseñanza y aprendizaje. Laurus, 13(23), 213-234, e-ISSN: 1315883X. Recuperado de:

https://www.redalyc.org/articulo.oa?id=76102311

Cisneros, A. (comp.). (2004). Manual de estilos de aprendizaje. DGB/DCA/12-2004. México: Secretaría de Educación Pública; Subsecretaría de Educación Media Superior. Recuperado de: https://biblioteca.pucv.cl/site/colecciones/manuales u/Manual Estilos de Aprendizaje 2004.pdf

Córdova, N., Oliveros, E., \& Villena, M. (2013). La matemática superior y modelo de congruencia educativa. Gaceta Sansana, 1(2), 17-31, eISSN: 1390-7840. Recuperado de:

http://publicaciones.usm.edu.ec/index.php/GS/article/view/24

Córdova, N., Peña, R., \& Sarzosa, R. (2012). Elementos de innovación docente y su impacto en la mejora del aprendizaje: Clasificación de competencias Tuning dentro del modelo de congruencia educativa. Gaceta Sansana, 1(1), 50-57, e-ISSN: 1390-7840. 
Recuperado de:

http://publicaciones.usm.edu.ec/index.php/GS/article/view/36/56

Del Salto, M. (2015). Educación en valores: propuesta de una estrategia. Medisan, 19(11), 5018-5026, e-ISSN: 1029-3019. Recuperado de: http://www.medisan.sld.cu/index.php/san/article/view/533

Díaz, G., \& Guambi, D. (2018). La innovación: baluarte fundamental para las organizaciones. Innova Research Journal, 3(10.1), 212-229, eISSN: 2477-9024. Recuperado de:

https://revistas.uide.edu.ec/index.php/innova/article/view/843/866

Fernández, R. (2005). Evaluación Psicológica. Madrid, España: Ediciones Pirámide.

Figueroa, R. (2015). La evaluación educativa en la pedagogía de las ciencias naturales, enmarcada en las universidades venezolanas. Saber, 27(2), 310-323, e-ISSN: 1315-0162. Recuperado de: http://ve.scielo.org/scielo.php?script=sci arttext\&pid=S1315$\underline{01622015000200013}$

García, T., Rodríguez, C., González-Castro, P., Álvarez-García, D., \& González-Pienda, J. (2016). Metacognición y funcionamiento ejecutivo en Educación Primaria. Anales de Psicología, 32(2), 474483, e-ISSN: 0212-9728. Recuperado de:

https://www.redalyc.org/articulo.oa?id=16745250019

Gardner, H. (2015). Inteligencias Múltiples: La teoría en práctica. Barcelona, España: Ediciones Paidós.

Ministerio de Educación del Perú (2014). 15 Buenas prácticas docentes: Experiencias pedagógicas premiadas en el I Concurso Nacional de Buenas Prácticas Docentes. Primera edición, № 2014-09035. Lima, Perú: Prysma S.A.C. Distribuidora Importadora. Recuperado de: https://www.minedu.gob.pe/buenaspracticasdocentes/pdf/pub4.pdf Ministerio de Educación (2012). Título VI: De la evaluación, calificación y 
promoción de los estudiantes. Capítulo I: De la evaluación de los aprendizajes. Reglamento General a la Ley Orgánica de Educación Intercultural. Quito, Ecuador: Dirección Nacional de Normativa Jurídico Educativa.

Ministerio de Educación del Ecuador (2011). Pedagogía y didáctica. Segunda edición, ISBN: 978-9978-92-816-5. Quito, Ecuador: Centro Gráfico Ministerio de Educación - DINSE.

Navarro, N., Falconí, A., \& Espinoza, J. (2017). El mejoramiento del proceso de evaluación de los estudiantes de la Educación Básica. Universidad y Sociedad, 9(4), 58-69, e-ISSN: 2218-3620. Recuperado de: https://rus.ucf.edu.cu/index.php/rus/article/view/665

Ruiz, R., \& Tesouro, M. (2013). Beneficios e inconvenientes de las nuevas tecnologías en el aprendizaje del alumno. Propuestas formativas para alumnos, profesores y padres. Revista Educación y Futuro Digital, 7, 17-27, e-ISSN: 1695-4297. Recuperado de:

http://hdl.handle.net/11162/118963

Suárez, J., Maiz, F., \& Meza, M. (2010). Inteligencias múltiples: una innovación pedagógica para potenciar el proceso enseñanza aprendizaje. Investigación y Postgrado, 25(1), 81-94, e-ISSN: 13160087. Recuperado de:

https://www.redalyc.org/articulo.oa?id=65822264005

Tourón, J., Santiago, R., \& Díez, A. (2014). The Flipped classroom: Cómo convertir la escuela en un espacio de aprendizaje. Navarra, España: Grupo Océano.

Vázquez-Chaves, A. (2015). La metacognición: Una herramienta para promover un ambiente áulico inclusivo para estudiantes con discapacidad. Revista Electrónica Educare, 19(3), 1-20, e-ISSN: 14094258. Recuperado de:

https://www.redalyc.org/articulo.oa?id=194140994007 


\section{Christian Virgilio Guerrero Salazar e-mail: chguerrero.ascaped@gmail.com}

Nacido en Guayaquil, Ecuador, el 8 de julio del año 1977.

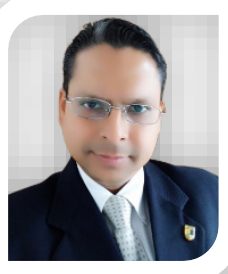
Licenciado en Ciencias de la Educación, Especialización FIMA de la Facultad de Filosofía, Letras y Ciencias de la Educación de la Universidad de Guayaquil (UG); tengo una experiencia docente de más de 20 años colaborando con distintas instituciones de educación media y superior de la ciudad de Guayaquil, y actualmente me desempeño como docente y gestor pedagógico en la Unidad Educativa Bilingüe Torremar; soy director pedagógico de ASCAPED, empresa dedicada a la capacitación y asesoría educativa; he colaborado durante varios años con diferentes Universidades, fundaciones y editoriales ecuatorianas como capacitador docente, asesor pedagógico, investigador, etimólogo y Metodólogo; soy consultor en el área pedagógica e instructor de cursos de formación de la Organización de Estados Iberoamericanos (OEI); he participado en varios eventos nacionales e internacionales como ponente; cuento con variadas publicaciones en revistas indexadas. 


\section{Yeimer Prieto López}

e-mail: yeiprietolopez@gmail.com

Nacido en Holguín, Cuba, el 1 de marzo del año 1981.

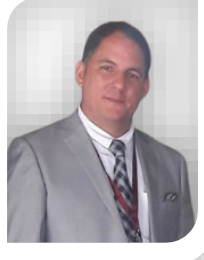

PhD. Doctor en Ciencias de la Educación; Licenciatura en

Educación; Profesor Titular de la Universidad Espíritu Santo (UEES) en la Facultad de Artes Liberales; Profesor del Máster en Gestión Educativa de la UEES; Docente y auditor académico en la Unidad Educativa Bilingüe Torremar; he participado en varios eventos nacionales e internacionales como ponente; tengo variadas publicaciones en revistas indexadas; he trabajado durante más de quince años como docente universitario y he tenido diferentes responsabilidades, como director de la Formación Doctoral en Cultura Física y Deportes de la Universidad de Holguín (UHO), Cuba; he participado en más de veinte congresos nacionales e internacionales de Pedagogía, Educación y Didáctica; soy tutor de varias tesis doctorales en la Universidad Nacional de Rosario (UNR), Argentina; hasta el momento he formado a tres Doctores en Ciencias en el Tribunal de Ciencias Pedagógicas de Cuba. 